\title{
Bioelectronic Sniffer (Biosniffer) Based on Enzyme Inhibition of Butyrylcholinesterase for Toluene Detection
}

\author{
Hirokazu Saito, Yuki Suzuki ${ }^{1}$, Tomoko Gessei ${ }^{1}, K_{\text {Kumiko Miyajima }}{ }^{1}$, \\ Takahiro Arakawa ${ }^{1}$ and Kohji Mitsubayashi, ${ }^{1, *}$ \\ Department of Mechanical Engineering, Tokyo National College of Technology, \\ 1220-2 Kunugida-machi, Hachioji-shi, Tokyo 193-0997, Japan \\ 'Institute of Biomaterials and Bioengineering, Tokyo Medical and Dental University, \\ 2-3-10 Kanda-Surugadai, Chiyoda-ku, Tokyo 101-0062, Japan
}

(Received Novermber 18, 2013; accepted February 19, 2014)

Key words: biosniffer, biosensor, toluene, enzyme inhibition, butyrylcholinesterase, choline oxidase

A new bioelectronic sniffer (biosniffer) for toluene was developed with butyrylcholinesterase (BuChE) and choline oxidase (COD), and evaluated for its performance. The toluene was measured as the effect of inhibitor on the enzyme activity of BuChE. The biosniffer device consisted of an enzyme-immobilized biosensor and a flow cell for the measurement of gaseous substances. The biosensor for toluene in the liquid phase was constructed using a Clark-type dissolved oxygen electrode and a BuChE and COD coimmobilized membrane. After evaluating the biosensor's performances with choline and butyrylcholine, the characteristics of the biosniffer for toluene were evaluated in the gas phase. As a result, the signal intensity of the biosniffer with $750 \mu \mathrm{mol} / 1$ butyrylcholine decreased upon application of toluene vapor and reached a steady-state current, thus revealing the concentration of toluene in the gas phase. The changes in output current of the biosniffer were found to be related to the toluene concentration over the range from 5 to $100 \mathrm{ppm}$ with a correlation coefficient of 0.997 ( $n$ $=5$ ). The calibration range of the biosniffer for gaseous toluene covered the permissible concentration of toluene (50 ppm) as the threshold limit value (TLV) prescribed by the American Conference of Governmental Industrial Hygienists (ACGIH). The biosniffer (gas-phase biosensor) based on enzyme inhibition was effectively used for the measurement of toluene vapor.

${ }^{*}$ Corresponding author: e-mail: m.bdi@tmd.ac.jp 


\section{Introduction}

Toluene is an aromatic hydrocarbon and is mainly used as a solvent in the production of paints, paint thinners, and adhesives. ${ }^{(1)}$ However, long-term exposure to toluene causes dysfunction of the central nervous system, such as constriction of the visual field, ataxia, and amnesia, since it inhibits the enzyme activity of acetylcholinesterase, which catalyzes the hydrolysis of acetylcholine, one of the neurotransmitters in the central nervous system Thus, the maximum permissible concentration [threshold limit value (TLV)] of toluene is prescribed by the American Conference of Industrial Hygienists (ACGIH) as $50 \mathrm{ppm}$ in air for $8 \mathrm{~h} .{ }^{(2)}$ Therefore, a sensitive, simple, and reproducible method of detecting gaseous toluene is required to maintain environmental safety for a working person and his surroundings. Some methods of toluene detection have been reported, such as the use of a detection tube, ${ }^{(3)}$ gas chromatography, ${ }^{(4)}$ and semiconductor sensors. ${ }^{(5)}$ However, each method has its advantages and disadvantages. Although the detection tube method ${ }^{(3)}$ is simple and quick, it cannot be used for continuous measurement. Gas chromatography(4) is sensitive and specific but it is costly and not designed for field operations. Semiconductor gas sensors are not so selective for their target chemicals in the gas phase. To solve these problems, some biosniffers (biochemical gas sensors) have recently been constructed using bio-electronic or bio-optic techniques..$^{(6-13)}$ The biochemical sensors with enzyme proteins show good selectivity for their target volatile chemicals because of enzyme specificity. ${ }^{(14)}$

On the other hand, many types of biosensor based on enzyme inhibition have been developed and reported.(15-17) Moreover, toluene inhibits the catalytic reaction of butyrylcholinesterase. ${ }^{(18,19)}$ In this research, we have constructed a biosensor and a biochemical gas sensor (biosniffer) for toluene on the basis of the inhibition of enzyme activity. The sensors were fabricated with an oxygen electrode and an enzymeimmobilized membrane. The performances of the biosensor and biosniffer were evaluated in liquid and gas phases, respectively.

\section{Experimental Section}

\subsection{Materials}

A toluene biosensor in the liquid phase was constructed using an enzymeimmobilized biosensor with a commercially available Clark-type dissolved oxygen electrode (Oriental Yeast Co., Ltd., Tokyo, Japan). A flow cell was incorporated with the toluene biosensor for the measurement of gaseous toluene. An enzyme membrane was prepared from butyrylcholinesterase (BuChE; E.C.3.1.1.8, C7512, 1200 units, from Pichia pastoris, Sigma, Tokyo, Japan), choline oxidase (COD; E.C.1.1.3.17, C7512, 1000 units from Alcaligenes sp., Funakoshi Co., Ltd., Tokyo, Japan), photo-crosslinkable polyvinyl alcohol containing stilbazolium groups [PVA-SbQ: Type: SPP-H-13 (Bio), Toyo Gosei Co., Ltd., Tokyo, Japan], ${ }^{(20)}$ and a dialysis membrane (part No. 157-0144-02, thickness: $15 \mu \mathrm{m}$, Technicon Chemicals Co., S.A., Oecq, Belgium). The COD was first desalted with a desalting column (Ultrafree-MC; UFC3LGC25, MW10000, Millipore, Tokyo, Japan) so as not to coagulate with the PVA-SbQ. A phosphate buffer solution (100 $\mu \mathrm{mol} / \mathrm{l}, \mathrm{pH}$ 8.0) was prepared by mixing disodium hydrogen phosphate and potassium di-hydrogen phosphate (197-02865 and 169-04245, respectively, Wako Pure Chemical 
Industries, Ltd., Osaka, Japan). Choline (choline chloride, B2753, Kantokagaku Co., Ltd., Tokyo, Japan) and butyrylcholine (butyrylcholine chloride, B2753, Sigma, Tokyo, Japan) solutions were prepared for the evaluation of the sensor characteristics.

To measure the output of the biosniffer for toluene, a batch flow measurement system was constructed using a personal computer, a computer-controlled potentiostat (Model 1112, BAS Inc., Tokyo, Japan), an analogue-to-digital (A/D) converter (ADC-16, Pico Technology Co., Ltd., Cambridgeshire, UK), and a gas chamber $\left(41 \times 41 \times 30 \mathrm{~cm}^{3}=\right.$ $0.05 \mathrm{~m}^{3}$ ). A peristaltic pump (Type: MP-3N, Tokyo Rikakikai Co., Ltd., Tokyo, Japan) circulated the phosphate buffer solution between a carrier reservoir and the flow cell of the biosniffer.

\subsection{Toluene biosensor based on inhibition of butyrylcholinesterase}

The toluene biosensor was constructed using the Clark-type dissolved oxygen electrode and the enzyme-immobilized membrane. Figure 1 shows the process of immobilizing the enzymes into the porous dialysis membrane and a schematic diagram of the toluene biosensor. The enzyme mixure of BuChE $\left(1.6\right.$ units $\left.\cdot \mathrm{cm}^{2}\right)$ and desalted COD $\left(2.1\right.$ units $\left.\cdot \mathrm{cm}^{2}\right)$ with PVA-SbQ $(100 \mathrm{mg})$ and the phosphate buffer $(100 \mu \mathrm{l})$ was coated onto the dialysis membrane. After desiccating in the dark below $2{ }^{\circ} \mathrm{C}$ for $1 \mathrm{~h}$, the enzyme-coated membrane was irradiated with fluorescent light for $30 \mathrm{~min}$ for photocrosslinking of the PVA-SbQ. The BuChE and COD coimmobilized membrane $\left(5 \mathrm{~mm}^{2}\right)$ was placed onto the sensing area of the oxygen electrode covered with a supporting nylon mesh net and secured with a silicone O-ring. The sensing area of the electrode was moistened with the buffer solution to maintain the enzyme activity until it was used for the measurement of toluene.

The enzyme reactions of BuChE and COD are shown as chemical equations in Fig. 2. BuChE catalyzes the hydrolysis of butyrylcholine with the production of choline and butyric acid. Then, choline as the BuChE enzymatic product is oxidized to betaine by COD. Therefore, the sensor can be used to measure butyrylcholine by detecting the oxygen consumption by COD. However, if toluene exists in the reaction system, toluene inhibits the enzymatic activity of BuChE. As a result, a reduction of oxygen consumption reflects the concentration of toluene in the liquid or gas phase.

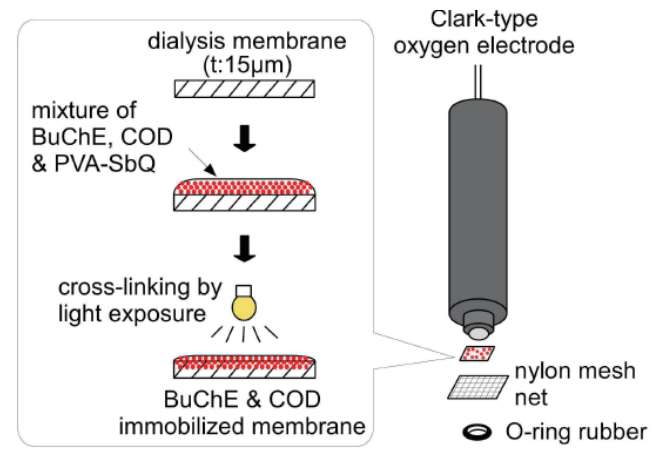

Fig. 1. (Color online) Fabrication procedure of an enzyme-immobilized membrane and biosensor for toluene. 


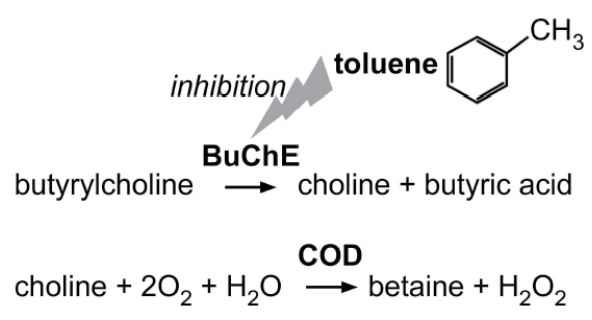

Fig. 2. Principle of toluene measurement using enzymatic inhibition of butyrylcholinesterase.

To evaluate the characteristics of the toluene biosensor, we first verified the COD reaction by measuring the sensor response to the choline solution. After the tip of the biosensor was immersed in $50 \mathrm{ml}$ of the buffer solution, the choline solution was dropped into the buffer solution agitated by a stirrer. The biosensor response was monitored at various concentrations of the choline solution. The output current of the biosensor was measured using a computer-controlled potentiostat, and stored on a hard disk of a personal computer via the analogue-to-digital converter for later analysis.

In the next experiment, the BuChE and COD coimmobilized sensor was used for the butyrylcholine measurement to verify the catalytic reactions of the two enzymes (Fig. 1) and to evaluate the calibration characteristics. The tip of the biosensor was immersed in the phosphate buffer solution, and then the standard solution of butyrylcholine was dropped into the buffer solution. The responses of the biosensor were examined at various concentrations of the butyrylcholine solution. On the basis of this experiment, the appropriate concentration of butyrylcholine for use in toluene measurement was determined.

\subsection{Bioelectronic sniffer for toluene in gas phase}

A bioelectronic gas sensor (biosniffer) with the BuChE and COD coimmobilized membrane was used to measure toluene in the gas phase. Figure 3 shows a schematic diagram of the biosniffer for the measurement of toluene vapor. The sniffer device consisted of the BuChE and COD coimmobilized biosensor and a flow cell with gas and liquid compartments separating a diaphragm. The flow cell was assembled with polytetrafluoroethylene (PTFE) pipe, a ringed rubber, a silicone sheet, a hydrophobic porous PTFE diaphragm membrane (pore size: 30-60 $\mu \mathrm{m}$, thickness: $0.20 \mathrm{~mm}$, ZITEX, NORTON KK, Nagano, Japan), and an acrylic cock. The biosensor with the enzyme membrane was inserted into the PTFE pipe body and supported with the O-ring. The flow cell between the PTFE pipe and the acrylic cock was clamped and fixed with a vise. The sensing tip of the enzyme biosensor was placed into the flow cell to touch the PTFE diaphragm membrane. In this biosniffer, the gaseous toluene from the gas compartment through the porous PTFE diaphragm membrane was detected on the basis of the inhibition of the BuChE reaction in the biosensor. 


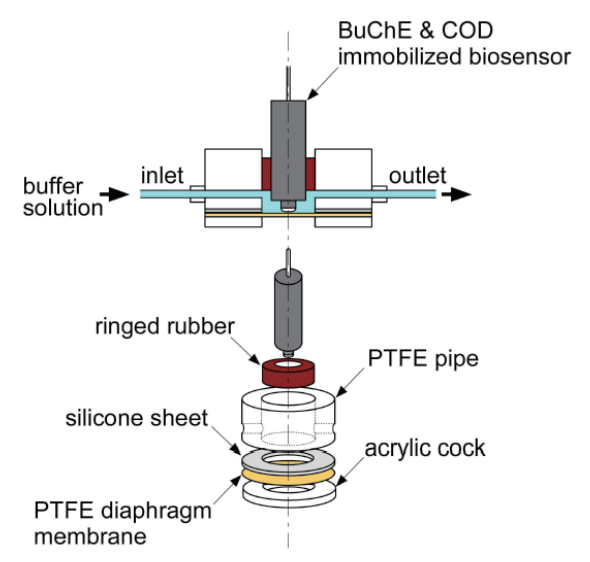

Fig. 3. (Color online) Schematic of a bioelectronic gas sensor (biosniffer) consisting of the enzyme-immobilized biosensor and a flow cell for gaseous toluene.

The phosphate buffer solution with butyrylcholine flowed into the liquid compartment of the flow cell. To measure the gaseous toluene as the inhibitor, the phosphate buffer solution was circulated in the cell to supply the appropriate concentration of butyrylcholine and dissolved oxygen as the enzyme substrates, and to remove the enzyme products (i.e., butyric acid, betaine, hydrogen peroxide) and surplus toluene that were diffused through the diaphragm membrane and the enzyme membrane at the tip of the biosensor from the gas compartment.

Figure 4 shows the experimental setup of toluene measurement in the gas phase. The toluene biosniffer described above was set inside the gas chamber. A peristaltic pump circulated the butyrylcholine solution between the carrier reservoir and the biosniffer at a constant flow rate of $1.0 \mathrm{ml} / \mathrm{min}$. The concentration of the butyrylcholine solution was determined at the level of $750 \mu \mathrm{mol} / \mathrm{l}$ from the examination of biosensor responses. After the sensor output was stabilized, the toluene solution was dropped on a volatile plate set in the gas chamber. Gaseous toluene vaporized immediately from the plate set and filled the chamber using a fan. Then, the toluene molecules reached the enzyme membrane of the biosensor and inhibited the BuChE catalytic reaction. The changes in biosniffer output were monitored using a personal computer via the potentiostat and $\mathrm{A} / \mathrm{D}$ converter.

\section{Results and Discussion}

\subsection{Evaluation of biosensor for choline and butyrylcholine}

The performance of the toluene biosensor for choline and butyrylcholine solutions was assessed prior to use for toluene measurement. The output current of the biosensor increased rapidly to the steady state immediately upon the addition of the 


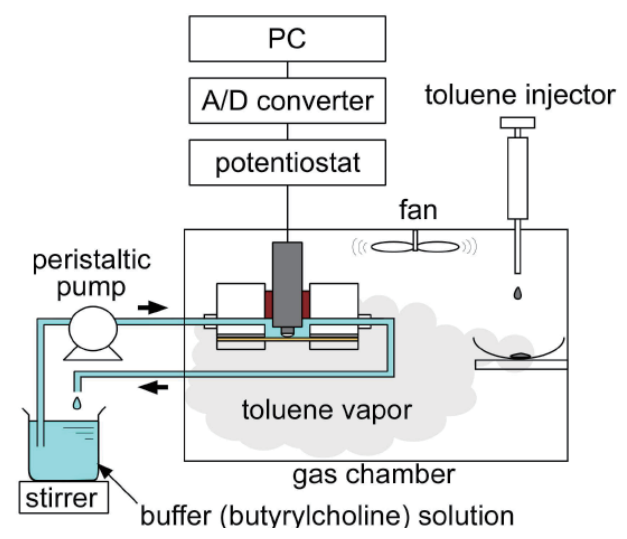

Fig. 4. (Color online) Measurement system for toluene in gas phase.

standard choline solution. The response time to reach $90 \%$ of the steady output was approximately $36 \mathrm{~s}$. The output of the biosensor was linearly related to the choline concentration over a range of $2.5-400 \mu \mathrm{mol} / \mathrm{l}$, with a correlation coefficient of 0.997 deduced by regression analysis as shown by eq. (1),

$$
\text { Sensor output }(\mathrm{nA})=0.0245+2.266[\text { choline }(\mu \mathrm{mol} / 1)] \text {. }
$$

The steady-state output signal over $400 \mu \mathrm{mol} / \mathrm{l}$ of choline concentration would indicate that the dissolved oxygen was saturated in the solution.

In the next experiments, the performance of the biosensor for the butyrylcholine solution was estimated. The calibration curve of the sensor output for the butyrylcholine solution is shown in Fig. 5. The inset of Fig 5 shows the typical responses of the biosensor to various concentrations of the butyrylcholine solution. The sensor output increased rapidly to the steady state following the addition of the standard butyrylcholine solution. The response time to reach $90 \%$ of the steady output after applying the butyrylcholine solution was approximately $44 \mathrm{~s}$. The output of the biosensor was linearly related to the butyrylcholine concentration over a range of $2.5-750 \mu \mathrm{mol} / 1$, with a correlation coefficient of 0.994 deduced by regression analysis as shown by eq. (2),

$$
\text { Sensor output }(\mathrm{nA})=0.0238+1.050[\text { butyrylcholine }(\mu \mathrm{mol} / \mathrm{l})] \text {. }
$$

The sensor performance was reproducible over multiple measurements, showing a coefficient of variation of $4.21 \%$ calculated from the steady-state output (average, $0.664 \mu \mathrm{A}$; standard deviation, $0.0279 \mu \mathrm{A} ; n=10$ ) at the $600 \mu \mathrm{mol} / 1$ concentration of butyrylcholine solution. As a result, the butyrylcholine concentration was adjusted to $750 \mu \mathrm{mol} / \mathrm{l}$ as the appropriate concentration in the buffer solution to use for the measurement of toluene as the $\mathrm{BuChE}$ inhibitor. 


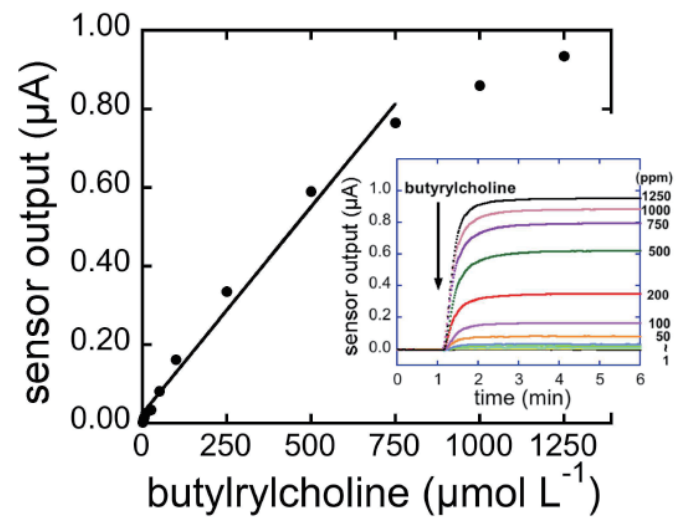

Fig. 5. (Color online) Calibration curve of biosensor for butyrylcholine in butyrylcholine solution. Inset: typical responses of biosensor to various concentrations of butyrylcholine solution.

\subsection{Characteristics of biosniffer for toluene vapor}

Figure 6 shows the typical responses of the biosniffer when the toluene solution was applied into the gas chamber, as the toluene concentration would be 5, 25, and $50 \mathrm{ppm}$. As the figure indicates, after the toluene application into the gas chamber, the sensor output decreased gradually and reached the steady-state level corresponding to the toluene concentration. The response times to reach $90 \%$ of the steady state after applying the toluene solution were approximately $3.8,7.8$, and $9.6 \mathrm{~min}$ at 5, 25, and $50 \mathrm{ppm}$ toluene, respectively. It seemed that the extension of response time corresponding to the toluene concentration mainly depended on the time required to volatilize completely and diffuse uniformly the toluene in the gas chamber.

The calibration curve of the biosniffer for gaseous toluene is illustrated in Fig. 7. The output of the biosniffer was linearly related to the toluene concentration over a range of 5-100 ppm, with a correlation coefficient of 0.997 deduced by regression analysis as shown by eq. (3),

$$
\text { Change in output }(\mu \mathrm{A})=-0.026+0.046 \log \text { [toluene }(\mathrm{ppm})] \text {. }
$$

The calibration range of the biosniffer covers the permissible concentration of toluene [time weighted average (TWA), $50.0 \mathrm{ppm}$ ] prescribed by ACGIH. Therefore, this biosniffer can be used for a simple and facile measurement of toluene vapor in a working environment such as a printing factory and chemical plant.

The sensor performance was reproducible over multiple measurements, showing a coefficient of variation of $7.28 \%$ calculated on the basis of the steady-state output (average, $55 \mathrm{nA}$; standard deviation, $4 \mathrm{nA} ; n=5$ ) for the measurement of $50 \mathrm{ppm}$ toluene in the gas phase. 

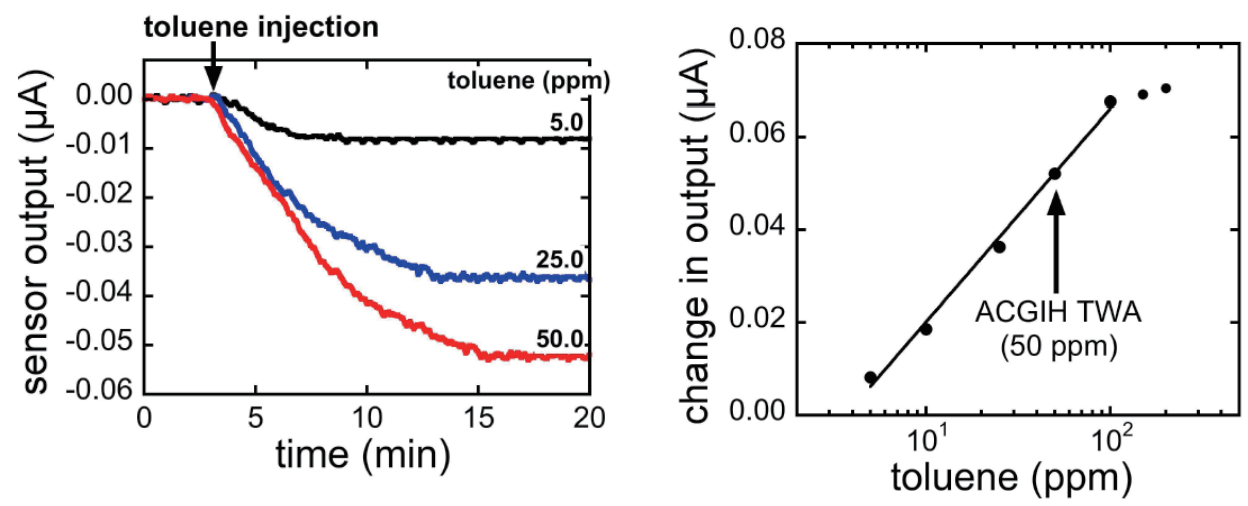

Fig. 6 (left). (Color online) Typical responses of biosniffer to various concentrations of toluene vapor.

Fig. 7 (right). Calibration curve of biosniffer for toluene in gas phase (arrow: permissible concentration of toluene prescribed by ACGIH, 50 ppm).

\section{Conclusions}

A bioelectronic sniffer (biosniffer) for toluene in the gas phase was developed on the basis of the inhibition of BuChE activity. The toluene biosniffer was assembled with the $\mathrm{BuChE}$ and COD coimmobilized electrode and the flow cell with a separating diaphragm between the gas and liquid compartments. Prior to the evaluation of the biosniffer, the characteristics of the biosensor were evaluated using standard solutions of choline and butyrylcholine, thus obtaining the calibration curves for choline $(2.5-400 \mu \mathrm{mol} / \mathrm{l})$ and butyrylcholine $(2.5-750 \mu \mathrm{mol} / 1)$. Then, gaseous toluene was measured as the current decrease of the biosniffer by the toluene inhibition from the original current to 750 $\mu \mathrm{mol} / \mathrm{l}$ butyrylcholine in the buffer solution. The calibration range for gaseous toluene was from 5 to $100 \mathrm{ppm}$, covering the permissible concentration of toluene (50 ppm) stipulated by ACGIH. The potential applications of the biosniffer using the enzyme inhibition principle include not only environmental analysis but also the conventional and quick detection of nerve gases such as organic phosphorous compounds (e.g., sarin gas).

\section{Acknowledgements}

This study was partially supported by the Japan Society for the Promotion of Science (JSPS) Grants-in-Aid for Scientific Research and the Ministry of Education, Culture, Sports, Science and Technology (MEXT) Special Funds for Education and Research "Advanced Research Program in Sensing Biology". 


\section{References}

1 Agency for Toxic Substance and Disease Registry (ATSDR). (2000) Toxicological Profile for Toluene (CAS \# 108-88-3). Atlanta, GA: U.S. Department of Health and Human Services, Public Health Service. (https://docs.google.com/viewer?url=http\%3A\%2F\%2Fwww.atsdr. cdc.gov\%2Ftfacts56.pdf).

2 American Conference of Governmental Industrial Hygienists (ACGIH): Documentation of the Threshold Limit Values (TLVs) and Biological Exposure Indices (BEIs) - Toluene. (2007) (https://www.osha.gov/dts/chemicalsampling/data/CH 272200.html).

3 B. R. Hubbard and L. Silverman: Arch. Ind. Hyg. Occup. Med. 2 (1950) 49.

4 G. Bieniek, S. Kurkiewicz, T. Wilczok, K. Klimek, L. Swiatkowska and A. Lusiak: J. Occup. Health 46 (2004)181.

5 Y. Zeng, T. Zhanga, L. Wang, M. Kang, H. Fan, R. Wang and Y. He: Sens. Actuators, B 140 (2009) 73.

6 L. Campanella, G. Favero and M. Tomassetti: Anal. Lett. 34 (2001) 855.

7 L. Campenella, R. Cocco, M. P. Sammartino and M. Tomassetti: Sci. Total Environ. 123 (1992) 1.

8 L. Campenella, R. Cocco and M. Tomassetti: J. Pharm. Biomed. Anal. 10 (1992) 741.

9 L. Campenella, G. Favero, M. P. Sammartino, M. Tomassetti and G. Visco: Anal. Chim. Acta 426 (2001) 235.

10 C. E. Efstathiou, E. P. Dlamandis and T. P. Hadjiioannou: Anal. Chim. Acta 127 (1981) 173.

11 J. L. Marty, K. Sode and I. Karube: Anal. Chim. Acta 228 (1989) 49.

12 A. A. Sileiman and Y. Xu: Electroanal. 4 (1998) 240.

13 V. N. Arkhypova, S. V. Dzyadevych, A. P. Soldatkin, A. V. EI'skaya, N. Jaffrezic-Renault, H. Jaffrezic and C. Martelet: Talanta 55 (2001) 919.

14 H. Yamaguchi(Ed): New Biotechnology (Keigaku publisher, Tokyo, 1987) p. 141.

15 M. D. L. Castro and M. C. Herrera: Biosens. Bioelectron. 18 (2003) 279.

16 A. Amine, H. Mohammadi, I. Bourais and G. Palleschib: Biosens. Bioelectron. 21 (2006) 1405.

17 L. Campanella, M. Achilli, M. P. Sammartino and M. Tomassetti: J. Electroanal. Chem. 321 (1991) 237.

18 J. Järv and M. Speek: Biochem. Biophys. Acta 706 (1982) 174.

19 P. Skládal and J. Krejčí: Collect. Czech. Chem. Commun. 61 (1996) 985.

20 Y. Hasebe, K. Oshima, O. Takise and S. Uchiyama: Talanta 42 (1995) 2079. 